Mots. Les langages du politique

$86 \mid 2008$

Toponymes. Instruments et enjeux

\title{
Fonctionnements sociolinguistiques de la dénomination toponymique
}

Henri Boyer

\section{(2) OpenEdition \\ Journals}

Édition électronique

URL : https://journals.openedition.org/mots/12962

DOI : $10.4000 /$ mots. 12962

ISSN : 1960-6001

Éditeur

ENS Éditions

Édition imprimée

Date de publication : 30 mars 2008

Pagination : 9-21

ISBN : 978-2-84788-129-5

ISSN : 0243-6450

Référence électronique

Henri Boyer, «Fonctionnements sociolinguistiques de la dénomination toponymique », Mots. Les langages du politique [En ligne], 86 | 2008, mis en ligne le 30 mars 2010, consulté le 22 avril 2022. URL: http://journals.openedition.org/mots/12962 ; DOI : https://doi.org/10.4000/mots.12962 


\section{Fonctionnements sociolinguistiques de la dénomination toponymique}

\section{Un toponyme peut être plus qu'un toponyme}

Qu'on l'appelle «dénomination toponymique» ou «dénomination choronymique» (Cyr et Nagúg Metallic, 1999), l'acte par lequel un nom propre est donné à un lieu - ville, région, pays... - est bien un acte ethnosocioculturel et même bien souvent un «acte politique» (Gendron, 2003, p. 55). On a parlé de sociotoponymie à propos de la démarche qui considère la toponymie «sous un aspect plus synchronique que diachronique», et surtout qui s'intéresse «moins [aux] préoccupations étymologiques qu'[à] l'évolution, la transformation ou l'apparition et la disparition de formes toponymiques» (Guerrin, 1999, p. 210). C'est cette démarche ouverte à la transdisciplinarité qui inspire les propos développés ici. On cherchera donc dans cette contribution à cerner dans sa dimension sociolinguistique cet acte de dénomination singulier ${ }^{1}$ et à en illustrer une des modalités.

On peut proposer la distribution fonctionnelle suivante de l'usage des toponymes, selon un modèle à trois entrées, avec bivalence au sein de la deuxième entrée :

\section{La nomination de l'espace}

Il ne fait pas de doute que ce fonctionnement socio-pragmatique du toponyme qui en fait un instrument de localisation est au principe même de l'acte toponymique, qui vise à s' «approprier [l'espace] », à «en faire du territoire» (Akin, 1999, p. 9). Cette localisation concerne tous les lieux, habités ou non : le tènement, le cours d'eau (on parle d' «hydronymie»), le relief (on parle d'«horonymie»), le hameau, la ville et sa voierie (on parle d' «odonymie»), le «(petit) pays », le département, la région, le Pays (Baylon et Fabre, 1982). Qu'il s'agisse de l'hydronyme Garonne, de l'odonyme Las Ramblas ou du toponyme

1. Il existe d'autres types de dénominations: patronymique, glossonymique, ethnonymique...

Université de Montpellier 3, ARSER / DIPRALANG, henri.boyer@univ-montp3.fr 
Portugal, il n'est question que de nomination localisante, d'un «choix dénotatif», même s’il convient de ne pas négliger les cas où la nomination «laisse passer un peu d'affectivité collective, lorsque par exemple tel toponyme, échappant aux règles du réseau, vient ponctuellement exprimer une peur et la manière d'exorciser cette peur par l'acte même qui la nomme » (Fabre, 1997, p.18-19). Etil fautégalement prendre en considération un certain nombre de cas pour lesquels le «dénotatif » s'adjoint du «connotatif » : une «plus-value toponymique» (Guerrin, 1999, p. 225) due à un investissement représentationnelethnosocioculturel (Boyer, 2003). On songe par exemple au toponyme Marseille et dans un autre registre à Poitiers ou encore à Oradour... De même, il existe un certain nombre de cas où le «dénotatif» même fait problème et est l'objet d'une polémique : il en va ainsi, par exemple, du toponyme Makedonias [Macédoine], choisi par la jeune République indépendante anciennement membre de la Yougoslavie, et contesté officiellement par l'état grec pour cause, selon lui, d'usurpation toponymique, ou pour des raisons autres de l'odonyme Avenida del Generalísimo Franco à Barcelone, durant le franquisme (nommé en catalan par les autochtones antifranquistes Avinguda Diagonal ou, sans parti pris sociolinguistique, la Diagonal), ou encore du toponyme communautaire Galicia contesté par un certain nombre de militants de la langue galicienne au profit de Galiza.

En bref, le fonctionnement socio-pragmatique primaire (nomination localisante) au principe de l'acte de dénomination toponymique n'est donc pas toujours observable à l'état pur, si l'on peut dire: le toponyme est souvent plus qu'un toponyme ${ }^{2}$. C'est même ce qui invite le sociolinguiste à se mêler de toponymie, sans pour autant prétendre à une compétence d'onomasticien.

\section{La symbolisation dans / parl'espace}

Il s'agit de distinguer en nommant. Il y a bien entendu une importante teneur identitaire dans ce fonctionnement. On doit distinguer deux sous-types.

\section{La promotion patrimoniale}

Il y a alors, comme on l'a dit, superposition de la nomination localisante et d'une emblématisation ou d'une mythification. Il s'agit d'une intervention toponymique qui célèbre un personnage (mort ou vivant), un évènement, un objet, un lieu... notoire et consensuel, du moins pour une partie non négligeable de la communauté concernée. Si l'emblématisation s'en tient à ces deux critères et tend à promouvoir, par exemple, l'image exemplaire d'un

2. Voir, à propos de la graphie d’une langue, Patric Sauzet, 1990, «La grafia es mai que la grafia », Amiras / Repères, $\mathrm{n}^{\circ}$ 21, Edisud. 
artiste populaire (place Charles Trenet) ou d'un homme politique (avenue Charles de Gaulle), d'un lieu de mémoire (rue de Verdun)..., la mythification ajoute à la notoriété et à l'exemplarité la transcendance historique, la sublimation, l'unanimité (Boyer, 2003; Amossy, 1991): on ne compte plus en France les rue Jean Moulin ou les place de la Révolution...

\section{L'affirmation identitaire}

Dans ce cas, la fonction identitaire prend ostensiblement le pas sur la fonction de localisation : au-delà de la nomination, il s'agit d'un acte de nature nettement politique ou plus spécifiquement glottopolitique. Il est question de revendiquer l'inscription du toponyme dans une communauté linguistique et / ou culturelle (nationale, régionale). On peut observer ce genre d'intervention (souvent lié à un positionnement régionaliste ou nationaliste) tout particulièrement dans des périodes de normalisation d'une langue jusqu'alors minorée parce que dominée, exclue entièrement ou pour l'essentiel de la communication publique institutionnelle. On songe par exemple à l'Espagne postfranquiste où les langues autres que le castillan ont récupéré, après l'adoption de la Constitution démocratique de 1978 et le vote de statuts d'autonomie par les communautés territoriales concernées, les usages sociolinguistiques confisqués par la langue dominante (et la dictature franquiste) : ce fut le cas dans les communautés autonomes de langue catalane (Catalogne, Communauté valencienne, Baléares), le Pays Basque (et une partie de la Navarre) et la Galice. Ainsi en Catalogne le Paseo de Gracia (castillan) de Barcelone est (re)devenu Passeig de Gracia (catalan) et la ville de Gerona a été renommée Girona, conformément à la norme toponymique catalane en vigueur dans la communauté autonome. Ce type de fonctionnement, forcément ostentatoire, peut prendre parfois l'allure d'une provocation : il en va ainsi lorsqu'en bordure d'une autoroute catalane, en direction de la France, un panneau indique les distances de quatre villes françaises, avec l'emploi de toponymes français pour trois d'entre elles - Narbonne, Toulouse, Montpellier - et d'un toponyme catalan pour la quatrième ville mentionnée, Perpinyà [Perpignan], capitale, dans l'interdiscours catalaniste, de la Catalunya Nord (le Roussillon)... Quant au Pays basque espagnol, on sait qu'il est redevenu Euskadi.

On peutévoquerégalementla redénomination des lieuxau Maghreb etparticulièrement en Algérie au lendemain de l'indépendance dans le cadre de la politique d'arabisation générale (Kahlouche, 1999, p. 183), à propos de la discrimination dont a été victime jusqu'à une date récente l'identité kabyle: on est même allé jusqu'à attribuer une origine arabe à des toponymes berbères comme pour «la ville de Maillot, par exemple, [...] redénommée M'chedallah, sens littéral probable "Qui compte sur Dieu", au mépris de la dénomination berbère usitée par les natifs de l'agglomération imechdalen, nom pluriel d'une 
espèce de fourmi rouge, singulier amechdal.» (Ibid, p. 187) On pourrait encore mentionner la mise en avant du toponyme Kanaky par le FLNKS en Nouvelle Calédonie, dans la perspective d'une accession à l'indépendance du territoire (Akin, 1999, p. 48-49; Dalhem, 1997). De fait, les conflits de dénomination liés à des antagonismes identitaires sont loin d'être exceptionnels.

\section{L’identification stéréotypique}

Il s'agit, d'une certaine façon, d'un cas d'emblématisation, le plus souvent (mais pas toujours) péjorante, éventuellement stigmatisante. C'est le processus d'antonomase qui prévaut ici (processus valable également pour la mythification, dont il a été question), processus par lequel un toponyme notoire (notoriété souvent due aujourd'hui à une surexposition médiatique) est instrumentalisé pour nommer un autre lieu qu'on vise à catégoriser (à étiqueter) par un stéréotype: on peut évoquer les cas de toponymes désignant quelques cités «à risques», diabolisées au cours des années quatre-vingts et quatre-vingt-dix (Les Minguettes par exemple) (Boyer et Lochard, 1998a). C'est un phénomène qui ne touche pas que des toponymes ayant émergé au fil de l'actualité des dernières années. On songe à des cas anciens et toujours disponibles comme Chicago, Waterloo... ou (à l'opposé, si l'on peut dire) Le Pérou, Venise.

Je voudrais dans ce qui suit illustrer, à travers deux cas de conflit d'ordre toponymique, le deuxième type de dénomination dont il vient d'être question et plus particulièrement l'affirmation identitaire. Le premier de ces deux cas concerne l'aventure politico-médiatique du toponyme Septimanie que Georges Frêche, nouveau président du conseil régional a tenté d'imposer en 2005 à l'opinion publique régionale comme substitut du toponyme en vigueur depuis 1960 (Languedoc-Roussillon), et de l'opposition manifestée, via la presse locale, contre ce qui est apparu comme un coup de force par une bonne partie de cette opinion publique. On observera un deuxième cas de conflit à propos d'une communauté autonome de l'état espagnol, en phase de reconquête sociolinguistique (et donc toponymique) : la Galice.

\section{"Vie et mort de la Septimanie $»^{3}$}

Àla suite du changement de majorité à la tête de la région Languedoc-Roussillon en 2004 et de l'élection du maire de Montpellier, Georges Frêche, à la présidence de cette même région, s'est ouverte une période de dénonciation de la présidence précédente, somme toute assez banale. Plus surprenante fut

3. Titre en page Eurorégion du quotidien L'Indépendant du $1^{\mathrm{er}}$ janvier 2006. 
la mise en cause de la dénomination de la région en question, compromis toponymique (consensuel) pour un territoire qui comprend essentiellement le Bas-Languedoc et la Catalogne française, au motif avancé par le nouveau président de région que «le nom de Languedoc-Roussillon [serait] totalement erroné sur le plan historique».

Une campagne publicitaire, jugée dispendieuse par ses détracteurs, a visé, durant l'été 2005, à diffuser massivement le toponyme présidentiel Septimanie. Cette volonté de redénomination ${ }^{4}$ et la campagne publicitaire autour de produits régionaux visant à imposer le label septimanien ont suscité de nombreuses réactions, pour la plupart d'opposition. Le quotidien régional Midi Libre (qui occupe sur le territoire languedocien concerné une position de quasi monopole), en septembre 2005, s'est saisi de la polémique, au travers d'une enquête et d'un appel à témoignages auprès de ses lecteurs (témoignages dont certains ont été publiés durant une semaine sur une page spécifique du journal). Il en est allé de même, avec semble-t-il encore plus d'acuité, pour le quotidien de Perpignan et du Roussillon L'Indépendant (appartenant au même groupe de presse). Ces initiatives, qui ont représenté incontestablement un succès médiatique et au total un gain de légitimation facilement acquis pour les deux organes de presse impliqués, ont mis en évidence un rejet très largement majoritaire de la redénomination toponymique. Georges Frêche s'est finalement incliné.

Bien que le toponyme Septimanie ait été avancé par le nouveau président du conseil régional tout de suite après son élection, c'est au printemps 2005 qu'est lancée publiquement par Georges Frêche la campagne visant à diffuser une nouvelle labellisation : Septimanie, destinée à promouvoir les produits de la région (fruits et légumes, coquillages, fromages...). Certains observateurs ont considéré qu'il ne s'agissait là que d'une nouvelle "griffe», sans plus. Mais bien vite les intentions réelles du président se sont manifestées plus clairement et d'aucuns se sont inquiétés de la marginalisation, dans la nouvelle communication de la région, du toponyme officiel Languedoc-Roussillon, maintenu cependant puisque étant le seul légal. Du reste, la livraison de mai 2005 ( $n^{\circ} 8$ ) du nouveau mensuel du conseil régional, ayant précisément pour titre Septimanie, annonce en couverture un dossier consacré à la "Naissance d'une marque » - «Septimanie. La griffe du soleil»-, dossier développé en pages intérieures (p. 6-8), qui présente le toponyme en question comme une "marque ombrelle» (associée au nouveau logotype de la région, où ne figurent désormais ni la croix occitane ni les couleurs du drapeau catalan, mais un assemblage stylisé de sept soleils). Le dossier célèbre le «volontarisme [...] de la région » et annonce une campagne

4. Assez curieuse, car le choix du toponyme officiel des collectivités régionales est légalement du ressort du pouvoir central et non du pouvoir régional. 
de communication estivale pour «la promotion des filières engagées par les conventions de partenariat signées avec la région [...], fédérant des familles de produits sous la marque collective Septimanie. Ce sera le cas avec les jardins de Septimanie, les vergers de Septimanie, les ruchers de Septimanie... » (Septimanie, $\mathrm{n}^{\circ} 8, \mathrm{p} .7$ ). La campagne de promotion annoncée aura bien lieu durant l'été 2005 et son coût très élevé ne sera pas un argument négligeable dans les réactions hostiles aux visées de Georges Frêche. Car commencent à s'élever, en particulier du côté de Perpignan et du Roussillon (la composante catalane de la région), de nombreuses voix dénonçant comme un coup de force (d'aucuns n'hésiteront pas à qualifier Georges Frêche de «dictateur») la volonté de redénomination de la collectivité territoriale. L'Indépendant, qui relaie cette contestation, reçoit un abondant courrier. Plusieurs pages intitulées «Septimanie: des réactions en cascade » y sont consacrées 5 .

Compte tenu de la nature bi-identitaire de la région (quatre départements appartiennent à l'espace culturel occitan: l'Aude, le Gard, L'Hérault et la Lozère; un cinquième, les Pyrénées-Orientales, est constitué pour l'essentiel de la Catalogne française, revendiquée par les nationalistes catalans des deux côtés des Pyrénées comme composante à part entière des « Països Catalans»), il est évident que "le nom "Languedoc-Roussillon" est le fruit d'un compromis» (Bernard Rieu, L'Indépendant, 27 juillet 2005, p. 20), acquis officiellement en juin 1960. Comme le rappelle Rieu, « la spécificité des PyrénéesOrientales a [été] reconnue afin de rallier les Catalans, dont beaucoup étaient réticents. "Roussillon" a été accolé à "Languedoc" pour désigner la nouvelle région " (ibid). Il est donc indéniable que la mobilisation contre le toponyme Septimanie a été particulièrement importante en Roussillon ${ }^{6}$. Comme le résume le journaliste de $L^{\prime}$ 'ndépendant qui a suivi au sein de la rédaction cette polémique publique, «depuis que le mot “Septimanie" s'insinue doucement mais sûrement dans les brochures régionales ou dans les publicités émanant de la région, ce ne sont que protestations véhémentes. Il suffit de consulter les centaines de lettres qui alimentent depuis des mois le courrier des lecteurs de L'Indépendant pour mesurer à quel point ce glissement sémantique est rejeté par les populations du département des Pyrénées-Orientales» (L'Indépendant, 7 août 2005). Un maire du département, Christian Blanc, a été à la pointe de la contestation anti-Septimanie avec une affiche publicitaire humoristique prônant la vaccination contre la «septimaniole», cause de coliques... De même, les autonomistes du parti «Bloc Català» ont utilisé, sur un mode tout aussi humoristique, l'animal emblématique des Catalans, l'âne, sous la figure vengeresse du «burro masqué » (l'âne masqué).

5. Je remercie Christian Di Scipio, journaliste à L'Indépendant, pour les informations qu'il m'a communiquées.

6. D’aucuns ont observé le silence, jugé suspect, du mouvement occitan durant la polémique. 
Une manifestation importante (plusieurs milliers de personnes) à Perpignan le 8 octobre 2005 a été le point d'orgue de cette fronde catalane, manifestation dans laquelle se sont certes investis les opposants politiques à Georges Frêche et à la majorité de gauche du conseil régional, mais qui a rassemblé également une foule d'opposants «identitaires» à la redénomination. Cette manifestation a pourtant eu lieu alors qu'elle pouvait paraître sans objet, le président de la région ayant déclaré plusieurs jours auparavant au quotidien Midi Libre (du 21 septembre 2005) qu'il renonçait à changer le nom de la région.

Cette volonté de Georges Frêche de renommer la région (que nombre de ses détracteurs n'hésitèrent pas à mettre sur le compte d'une mégalomanie notoire) n'avait pas manqué de s'appuyer sur un argumentaire en bonne et due forme, à teneur à la fois historienne et économique, basé sur un savoirfaire communicatif éprouvé. Dans l'une des interventions scripturales qui font partie de la campagne de légitimation du nouveau toponyme, un dépliant de quatorze pages intitulé «La Septimanie. Un nom, une histoire, un projet»" dans lequel il est bien évidemment fait référence à l'histoire ("Septimanie: d'où vient ce nom?»), on apprend que l'appellation promue remonte... au $5^{\text {e }}$ siècle et que l'objectif est de "Renouer avec notre histoire». Le texte qui coiffe l'ensemble du discours tenu dans ce dépliant, signé par le « président de la région Languedoc-Roussillon / Septimanie» (titre: «Septimanie: une identité pour réussir»), fortement dialogique et polémique, condamne sans appel le toponyme en usage: "Le nom de Languedoc-Roussillon est totalement erroné sur le plan historique. Le Languedoc, c'est essentiellement la région toulousaine dont la capitale historique est Toulouse. Quant au Roussillon, les Catalans utilisent peu ce nom car il ne concerne que la plaine.» En sus du critère de légitimation géohistorique - discutable : le «Bas-Languedoc» est bien le «Languedoc » et le «Roussillon » est bien la traduction française d'un toponyme tout ce qu'il y a de plus catalan - est avancé un critère de notoriété non moins discutable: «Le Languedoc-Roussillon est une dénomination inexacte et très peu connue tant au plan national qu'international. " L'argument est en effet sans pertinence s'agissant du plan international, car ce qui vaut pour Languedoc-Roussillon vaut sans aucun doute pour Septimanie. Et sur le plan hexagonal, le toponyme Languedoc-Roussillon est tout autant connu que bien d'autres toponymes de collectivités régionales. Quant à l'argument historico-identitaire, il n'est guère plus convaincant: «Pour parler ensemble sur un même ton, il importait de revenir avant la division entre Occitans et Catalans qui s'est faite aux $\mathrm{x}^{\mathrm{e}}$ et $\mathrm{x}^{\mathrm{e}}$ siècles, lors de la dislocation de l'empire carolingien. Le nom de Septimanie, cité pour la première fois au ve siècle, s'est imposé par notre histoire.». Une conclusion sans surprise est ainsi posée:

7. Voir en annexe un extrait de ce document: l'intervention signée de Georges Frêche. 
«à travers ce nom ["Septimanie"], toute la région et ses habitants, les Septimaniens et Septimaniennes, sont ainsi représentés dans leur identité et leur diversité.»

Manifestement, les efforts de communication et d'argumentation n'ont pas été couronnés de succès, mais ils ont produit un abondant interdiscours auquel ne s'attendait sûrement pas Georges Frêche. Même si le président sortait affaibli d'une longue convalescence, il a mis au service de cette entreprise de contre-dénomination sa légendaire faconde. Il n'est pas douteux qu'il a sous-estimé la prégnance identitaire et le pouvoir de mobilisation d'une dénomination toponymique (surtout du côté catalan de la région) pourtant composite, mais désormais bien établie après plus de quatre décennies d'existence.

Le quotidien Midi Libre, dans des conditions de moindre opposition (la fibre identitaire étant en Languedoc sûrement moins sensible qu'en Catalogne), a rendu compte des réactions hostiles de l'opinion publique languedocienne à l'entreprise de redénomination, en sollicitant ses lecteurs et en lançant, sur un forum ouvert à cet effet, une enquête: "Septimanie ou Languedoc-Roussillon? " $^{8}$. Dans une chronique intitulée "Vos voix au chapitre », en date du 24 septembre 2005 (c'est-à-dire après que le président du conseil régional eut annoncé qu'il ne débaptiserait pas la région), le médiateur du journal, Olivier Clerc, dressait le bilan et tirait les enseignements du «dossier Septimanie». En invoquant l'abondant échange entre les lecteurs et leur journal qu'avaient suscité « les velléités d'impérialisme toponymique du président du conseil régional» ("une levée de boucliers»), il mettait en avant «une opinion presque unanime, souvent abrupte: non à l'abandon de l'appellation "Languedoc-Roussillon" pour une improbable "Septimanie" ». Pour le rédacteur en chef, cité par le médiateur (qui fait état de diverses évaluations au sein de la rédaction), « le projet de Georges Frêche a visiblement été ressenti comme une privation d'identité, une négation de la "langue d'oc" " [c'est moi qui souligne]. Et d'avouer: "On ne pensait pas qu'il existait une telle identité languedocienne.» Finalement, et «au-delà du recul du président du conseil régional», l'opération est tout bénéfice pour le quotidien: démonstration d'empathie et donc, gain de légitimation médiatique (Lochard et Boyer, 1998b). Ainsi, même s' «il y a le sentiment que notre dossier à épisodes a pu aider l'exécutif régional à "botter en touche" dans une affaire bien mal engagée, il y a surtout la conviction d'un resserrement du lien avec

8. Site http://www.midilibre.com. Le texte invitant à participer à l'enquête était ainsi rédigé: «Depuis que Georges Frêche a sorti de son chapeau l'appellation Septimanie, avec l'idée de rebaptiser peut-être un jour le Languedoc-Roussillon, une véritable vague de protestation a déferlé sur la région. Midi Libre, qui a souvent abordé cette polémique, a voulu faire le tour de la question en publiant une enquête. Tous les aspects seront abordés: l'angle historique, le volet financier, la Catalogne fer de lance de la contestation et enfin les explications de Georges Frêche. Que pensez-vous de Septimanie? Exprimez-vous sur notre forum spécial!» 
nos lecteurs» (Midi Libre, 24 septembre 2005, p. 12). Dans la conférence de presse qu'il avait tenue le 23 septembre, consacrée certes à un autre sujet, mais aussi à sa « reculade» dans l'affaire "Septimanie », Georges Frêche avait déclaré, en commentant «l'enquête "honnête" de Midi Libre qui l'[avait] vivement intéressé »: "La démonstration est faite que les gens ne veulent pas que leur région change de nom. J'abandonne de manière définitive. " 9

Le président de la région Languedoc-Roussillon a depuis jeté son dévolu sur le toponyme Sud pour la promotion des productions agro-alimentaires de la région ("Sud de / South of France» et «Sud et Saveurs de France»). Séduisant même nombre de militants étonnés par un discours ostensiblement occitaniste, il s'est fait par ailleurs le chantre inspiré de la langue et de la culture occitanes lors d'une grande manifestation festive au cœur de Montpellier («Total Festum», le 21 octobre 2006) avec pour slogan «Le jour de l'OC est arrivé». Cette stratégie communicative bivalente consiste ainsi à dissocier une identification géo-économique (avec $S u d$ ) et une identication culturelle et linguistique (avec $O c)^{10}$.

\section{Le front toponymique d'une « bataille de la langue »}

En situation de domination d'une communauté sur une autre, ou d'un état sur l'une de ses composantes territoriales (ethnique, régionale ou nationale), on observe que la résistance et bien évidemment la reconquête identitaires se focalisent volontiers sur la dénomination toponymique: les noms de lieux sont alors investis d'une importante dimension symbolique. Ainsi la lutte pour le toponyme légitime est un chapitre proprement spectaculaire des résistances et reconquêtes communautaires: installer le toponyme conforme à la langue-culture jusque là minorée devient un enjeu politique de premier ordre, susceptible de mobiliser de nombreux acteurs. Il sera question dans cette dernière partie d'un épisode récent de ce front toponymique de la bataille de la langue ${ }^{11}$, épisode concernant une communauté autonome d'Espagne, la Galice, où est mise en œuvre la normalisation du galicien («langue propre», co-officielle dans la communauté avec le castillan, langue de l'État).

Le cas de la Galice est particulièrement intéressant parce que l'imaginaire communautaire des deux langues en contact y est complexe et pour l'essentiel, clivé. Si une minorité de Galiciens considèrent que leur communauté est une nation, et qu'elle doit en avoir les prérogatives intégrales, en particulier en matière de langue et de culture, une majorité n'adhère pas à cette vision des choses, même si un sentiment identitaire la conduit à se percevoir

9. Compte rendu de Midi Libre du 24 septembre 2005, p. 23 ; c'est moi qui souligne.

10. Par ailleurs, le mensuel de la région a depuis pris le titre de Vivre en Languedoc-Roussillon...

11. Titre de l'ouvrage d'un militant catalaniste: Pius Pujades, 1988, La batalla de la llengua. Converses amb Francesc Ferrer i Gironés, Barcelona, Xarxa Cultural. 
comme différente de l'ensemble espagnol. Ce clivage en recoupe un autre, qui concerne la langue galicienne : celle-ci est largement victime d'un stéréotypage négatif qui tend à en faire une langue de la ruralité, de la rusticité, du passé et de l'inculture, en même temps qu'un autre stéréotypage tend à en faire une langue patrimoniale dont le passé mérite d'être célébré, et une caractéristique identitaire importante. Ce stéréotypage ambivalent est la manifestation la plus flagrante de l'existence d'un conflit diglossique conforté par une prégnante idéologie diglossique (Boyer, 2005 et 2007). Il engendre dans à peu près toutes les strates sociales de la communauté des attitudes bien identifiées dans une telle configuration sociolinguistique comme la stigmatisation de la langue dominée, ici le galicien, et l'autodénigrement de la part de ses usagers. Le castillan, langue de la ville, de la distinction sociale, bien que n'ayant pas une position hégémonique du point de vue démolinguistique (Siguán, 1994 et 1999), est la langue dominante de la communauté. Sa représentation valorisante entrave la promotion du galicien, pourtant inscrite dans les perspectives officielles du statut d'autonomie de la Galice (adopté en 1981) et de la loi de normalisation linguistique votée par le Parlement galicien en 1983 (Alén Garabato et Boyer, 1997). La politique linguistique institutionnelle, précisément à cause d'un volontarisme très modéré de la part du parti espagnol de droite qui a gouverné la Galice durant plus d'une décennie (et bien que le président du gouvernement autonome, M. Fraga, ait su tirer parti d'une pratique personnelle courante de la «langue propre»), évidemment sensible à l'état de l'imaginaire collectif des langues dont il vient d'être question, n’a pas été à la hauteur des attentes des militants de la langue galicienne. Ces derniers ont donc dû s’organiser, depuis la société civile, en contre-pouvoir glottopolitique. C'est ainsi que s'est constitué le fer de lance associatif de la normalisation linguistique en Galice: A Mesa pola normalización lingüística (le Collectif pour la normalisation linguistique) dont l'engagement efficace n'a pas peu contribué à quelques avancées indéniables pour la défense et la promotion de la «langue propre» de la communauté12. Un épisode exemplaire de cette action revendicative de $A$ Mesa pola normalización mérite d'être présenté. Il convient de préciser auparavant la règle concernant la toponymie galicienne prévue par la Loi de normalisation linguistique de 1983. L'article 10 de cette loi indique en effet que :

12. La situation se complique du fait d'un conflit normatif concernant cette même «langue propre» qui sévit depuis l'adoption officielle de normes orthographiques, normes issues de la tendance "différentialiste » des militants de la langue et jugées trop suivistes à l'égard du castillan par les militants de l'autre tendance galéguiste, tenants d'une norme « réintégrationniste », basée sur le retour du galicien dans le giron lusophone. Ces deux grandes tendances, dont la deuxième présente une option radicale et une autre modérée (Alén Garabato 2000), se livrent un combat désolant au regard du vrai enjeu sociolinguistique: la conquête de la légitimité sociale du galicien dans de vastes secteurs de la communication sociale comme l'entreprise, le commerce, la justice... 
1. Les toponymes de Galice auront comme unique forme officielle la forme galicienne.

2. Il revient à la Xunta ${ }^{13}$ de Galice de déterminer les noms officiels des municipalités, des territoires, des centres de population, des voies de communication interurbaines et des toponymes de Galice.

3. Ces dénominations sont les seules légales à tous effets et la signalisation devra les respecter. La Xunta de Galice règlera la normalisation de la signalisation publique en respectant dans tous les cas les normes internationales adoptées par l'État ${ }^{14}$.

L'épisode en question concerne le toponyme Arousa, dont l'orthographe (castillanisée) a été jusqu'à sa normalisation: Arosa. Mais, au moins jusqu'en novembre 1996, il faut croire que le nouveau toponyme ne s'était pas imposé comme le seul légitime puisque la firme automobile SEAT, désirant baptiser l'un de ses nouveaux modèles (à l'instar des précédents : Toledo, Ibiza...) avec un toponyme d'Espagne avait choisi Arosa, du nom d'une Ría touristique de Galice. Devant la polémique déclenchée par A Mesa pola normalización, la direction de SEAT avait annulé la présentation de ce nouveau modèle sur le site choisi. Ce qui a engendré une autre polémique opposant les autorités locales, les hôteliers et restaurateurs (qui se sentaient lésés du point de vue des gains escomptés) aux fermes défenseurs du toponyme normalisé. L'argument de SEAT, selon lequel «Arosa sonne mieux dans les autres langues », n'a pas convaincu les opposants, pas plus que le fait qu'un nom donné à un précédent modèle de voiture de la firme (Ibiza: Esvissa en catalan) n’ait pas posé de problème. La polémique s'est prolongée durant l'année 1997. Les doléances réitérées de $A$ Mesa pola normalización n'ont pas ébranlé outre mesure la position de SEAT, considérée par certains commentateurs comme une « erreur commerciale ${ }^{15}$.

Contrairement au Movimento Defesa da Lingua (Mouvement Défense de la langue), la Mesa pola normalización n'a pas appelé au boycott de SEAT mais a sollicité la pression des citoyens de Galice face à ce qu'elle a dénoncé comme un "attentat contre notre identité ${ }^{16}$. Trois mille affiches et quarante mille cartes postales ainsi que des annonces dans la presse ont été utilisées comme vecteurs de la protestation, et il était demandé d'appeler la firme automobile par téléphone pour exiger le changement de nom (voir en annexe la reproduction de l'affiche).

13. Le gouvernement autonome.

14. Lei 31 /1983, do 15 de xuño, de normalización lingüística, Parlamento de Galicia (je traduis).

15. El Mundo du 18 décembre 1996. Les campagnes de la Mesa pola normalización ont trouvé un écho régulier dans la presse régionale (La Voz de Galicia, Le Correo Gallego), que j’ai consultée pour la période concernée.

16. Atentado contra a nosa identidade. 
Piètre concession : la firme d'origine espagnole décide de permettre aux acheteurs galiciens de choisir entre les deux orthographes du toponyme; tous les concessionnaires de Galice ont eu à cet effet à leur disposition un stock de plaques portant le nom de Arousa. On peut certes considérer qu'il s'agit là d'un demi-succès pour la Mesa pola normalización, qui s'est permis par ailleurs de fustiger l'équipe de communication de SEAT, qualifiée de «médiocre et peu cohérente ». On peut aussi considérer que la polémique a pu servir d'avertissement en direction d'autres entreprises, étrangères comme locales: un secteur considéré comme peu favorable à la normalisation linguistique (Formoso, 2004). Car l'enjeu de l'action de la Mesa pola normalización dépasse bien sûr le cadre des luttes circonscrites à telle ou telle revendication toponymique et s'inscrit dans une visée macrosociolinguistique de normalisation globale de la société galicienne, qui est loin d'être acquise.

Les conflits de dénomination toponymique tels que ceux qui viennent d'être observés, qu'ils soient de nature politique et ethnoculturelle comme celui qui a opposé Georges Frêche à l'opinion publique régionale du LanguedocRoussillon (surtout celle du Roussillon), ou plus spécifiquement de nature glottopolitique comme ceux concernant la normalisation du galicien, sont en définitive des conflits de légitimité qui révèlent les ressorts identitaires d'une communauté donnée. Dans les cas évoqués, le toponyme est bien un enjeu. Un enjeu pour une micro-région revendiquant une personnalité propre (la Catalogne française), sa latitude à afficher sa différence en refusant une dénomination exogène autoritaire. Un enjeu pour les militants d'une langue minoritaire au niveau de l'état (le galicien), jusqu'alors maltraitée par ce même état: ils témoignent de leur capacité à s'opposer aux actes (parfois illégaux) hostiles à la normalisation institutionnelle par une vigilance et une détermination sans faille.

\section{Références}

AkIN Salih, 1999, "Pour une typologie des processus redénominatifs», S. Akin éd., Noms et re-noms. La dénomination des personnes, des populations, des langues et des territoires, Rouen, Publications de l'Université de Rouen.

Alen Garabato Carmen, 2000, "Le galicien piégé par l'Histoire? La question de la norme », Lengas, $\mathrm{n}^{\circ} 47$.

Alen Garabato Carmen, Boyer Henri, 1997, "Les politiques linguistiques de deux communautés «historiques» d'Espagne: la Catalogne et la Galice», Mots. Les langages du politique, $\mathrm{n}^{\circ} 52$.

BAYLON Chistian, FABRE Paul, 1982, Les noms de lieux et de personnes, Paris, Nathan. BOUVIER Jean-Claude, GUILLON Jean-Marie, 2001, La toponymie urbaine. Significations et enjeux, Paris, L'Harmattan. 
BOYER Henri, 2003, De l'autre côté du discours. Recherches sur le fonctionnement des représentations communautaires, Paris, L'Harmattan.

- 2005, "Représentations linguistiques et idéologisation des conflits diglossiques», L. Jagueneau éd., Images et dynamique de la langue, Paris, L'Harmattan.

- 2007, "Le stéréotypage ambivalent comme indicateur de conflit diglossique», H. Boyer éd., Stéréotypage, stéréotypes. Fonctionnements ordinaires et mises en scène, Paris, L'Harmattan.

BOYER Henri, LAGARDE Christian, 2002, L'Espagne et ses langues, Paris, L'Harmattan.

BOYER Henri, Lochard Guy, 1998a, Scènes de télévision en banlieues (1950-1994), Paris, INA-L'Harmattan.

- 1998b, La communication médiatique, Paris, Le Seuil.

CYr Danielle E., Nagugwes Metallic Emmanuel, 1999, «Fantômes choronymiques de la dépossession », S. Akin éd., Noms et re-noms..., op. cit.

DAHLEM Jacqueline, 1997, "Des historiens à la recherche d'un consensus », Mots. Les langages du politique, $\mathrm{n}^{\circ} 53$.

DAROT Mireille, 1997, "Calédonie, Kanaky ou Caillou? Implicites identitaires dans la désignation de la Nouvelle-Calédonie », Mots. Les langages du politique, $\mathrm{n}^{\circ} 53$.

FABRE Paul, 1997, "Ce que la toponymie peut apporter à la... toponymie», Le Monde alpin et rhodanien, $2^{\mathrm{e}}-4^{\mathrm{e}}$ trimestres.

Formoso Gosende Valentina, 2005, A lingua galega no ámbito empresarial. Usos et representacións sociolingüísticas, Tese de doutoramento, Universidade de Santiago de Compostela, Departamento de Filoloxia galega.

GENDRON Stéphane, 2003, L'origine des noms de lieux en France, Paris, Errance.

GUERRIN Christian, 1999, "Les processus redénominatifs dans les noms de communes françaises depuis 1943. Étude socio-toponymique de la variation dans la nomenclature administrative », S. Akin éd., Noms et re-noms..., op. cit.

KAHLouche Rabah, 1999, "La dénomination/redénomination. Un lieu de conflit identitaire», S. Akin éd., Noms et re-noms..., op. cit.

Siguan Miquel, 1994, Opiniones y actitudes. Conocimiento y uso de las lenguas en España, Madrid, Centro de investigaciones sociológicas.

- 1999, Opiniones y actitudes. Conocimiento y uso de las lenguas, Madrid, Centro de investigaciones sociológicas. 